\title{
The Lie-Poisson Structure of Integrable Classical Non-Linear Sigma Models
}

\author{
M. Bordemann ${ }^{1, \star}$, M. Forger ${ }^{1}$, J. Laartz ${ }^{2, \star \star}$ and U. Schäper ${ }^{1}$ \\ 1 Fakultät für Physik der Universität Freiburg, Hermann-Herder-Strasse 3, \\ W-7800 Freiburg, Germany \\ 2 Department of Mathematics, Harvard University, 1 Oxford Street, Cambridge, MA 02138, USA
}

Received February 11, 1992

\begin{abstract}
The canonical structure of classical non-linear sigma models on Riemannian symmetric spaces, which constitute the most general class of classical non-linear sigma models known to be integrable, is shown to be governed by a fundamental Poisson bracket relation that fits into the $r-s$-matrix formalism for non-ultralocal integrable models first discussed by Maillet. The matrices $r$ and $s$ are computed explicitly and, being field dependent, satisfy fundamental Poisson bracket relations of their own, which can be expressed in terms of a new numerical matrix $c$. It is proposed that all these Poisson brackets taken together are representation conditions for a new kind of algebra which, for this class of models, replaces the classical Yang-Baxter algebra governing the canonical structure of ultralocal models. The Poisson brackets for the transition matrices are also computed, and the notorious regularization problem associated with the definition of the Poisson brackets for the monodromy matrices is discussed.
\end{abstract}

\section{Introduction}

During the last two decades, there has been great progress in understanding the structure of two-dimensional integrable field theories. Within the Hamiltonian approach [6], there have emerged new algebraic structures, notably the concept of Yang-Baxter algebras. These algebras appear, e.g., through the commutation relations of monodromy matrices when solving the models by the inverse scattering method.

At the classical level one begins by rewriting the equations of motion as a zero curvature condition, i.e., as the compatibility condition for a linear system with spectral parameter (Lax pair). In the Hamiltonian context one then studies the Poisson brackets

\footnotetext{
* Supported by the Deutsche Forschungsgemeinschaft, Contract No. Ro 864/1-1

$\star \star$ On leave of absence from Fakultät für Physik der Universität Freiburg, Supported by the Studienstiftung des Deutschen Volkes
} 
between $L$-matrices (the spatial part of the Lax pair). In the most common cases, this leads to a Lie-Poisson algebra of the form

$$
\{L(x, \lambda) \stackrel{\otimes}{,} L(y, \mu)\}=[r(\lambda, \mu), L(x, \lambda) \otimes 1+1 \otimes L(y, \mu)] \delta(x-y),
$$

with an antisymmetric $r$-matrix which is numerical, i.e., field independent, and which obeys the classical Yang-Baxter equation

$$
\left[r_{12}(\lambda, \mu), r_{13}(\lambda, \nu)\right]+\left[r_{12}(\lambda, \mu), r_{23}(\mu, \nu)\right]+\left[r_{13}(\lambda, \nu), r_{23}(\mu, \nu)\right]=0 .
$$

Theories for which the Poisson brackets between $L$-matrices are of this form are commonly called ultralocal [6] because the rhs of Eq. (1) contains only the delta function $\delta(x-y)$ but not its derivatives.

An important generalization of the above Lie-Poisson structure to certain nonultralocal models, namely those for which the rhs of Eq. (1) contains, apart from the delta function, its first derivative (i.e., a classical Schwinger term), has been developed by Maillet [14]. In his $r-s$-matrix approach, Eq. (1) is replaced by ${ }^{1}$

$$
\begin{aligned}
& \{L(x, \lambda) \stackrel{\otimes}{,} L(y, \mu)\}=-[r(x, \lambda, \mu), L(x, \lambda) \otimes 1+1 \otimes L(x, \mu)] \delta(x-y) \\
& +[s(x, \lambda, \mu), L(x, \lambda) \otimes 1-1 \otimes L(x, \mu)] \delta(x-y) \\
& -(r(x, \lambda, \mu)+s(x, \lambda, \mu)-r(y, \lambda, \mu)+s(y, \lambda, \mu)) \delta^{\prime}(x-y) \text {, }
\end{aligned}
$$

or equivalently, ${ }^{2}$

$$
\begin{aligned}
\{L(x, \lambda) \stackrel{\otimes}{,} L(y, \mu)\}= & r^{\prime}(x, \lambda, \mu) \delta(x-y) \\
& -[r(x, \lambda, \mu), L(x, \lambda) \otimes 1+1 \otimes L(x, \mu)] \delta(x-y) \\
& +[s(x, \lambda, \mu), L(x, \lambda) \otimes 1-1 \otimes L(x, \mu)] \delta(x-y) \\
& -\left(s(x, \lambda, \mu)+s(y, \lambda, \mu) \delta^{\prime}(x-y)\right.
\end{aligned}
$$

with an antisymmetric $r$-matrix and a symmetric $s$-matrix which, in general, depend explicitly on the fields of the theory. Some of the most important non-ultralocal models, such as the $O(N)$-sigma model $[4,12]$, the principal chiral model $[4,13]$ and the complex sine-Gordon model [14], are known to fit into this scheme.

In the present paper, we shall demonstrate that non-linear sigma models defined on Riemannian symmetric spaces, which constitute the general class of non-linear sigma models known to be integrable $[5,7]$, provide an explicit realization of this structure, with simple formulas expressing $r$ and $s$ as functions of the basic fields. In particular, the results of $[12,13]$ are special cases of the one obtained here.

At first sight, the fundamental Poisson brackets (3) or (4) are not particularly enlightening. They can however be brought into a more transparent form, which is also closer to the $r$-matrix approach used in classical mechanics [2], by passing from the $L$-matrix $L(x, \lambda)$ to the corresponding Lax operator $D(x, \lambda)$, defined as

$$
D(x, \lambda)=\frac{\partial}{\partial x}+L(x, \lambda) .
$$

Namely, the Poisson brackets of the D's are, by definition, the same as the ones for the $L$ 's, but $D$ being a differential operator, the inhomogeneous classical Schwinger terms on the rhs of Eqs. (3) or (4) can be absorbed into commutators (the commutator

1 Our $L$ corresponds to $-L$ in [14]

2 The equivalence of Eqs. (3) and (4) follows from the identity (6) given below 
of a $D$ with a delta function produces, among other things, the derivative of a delta function). Concretely, it follows directly from the identity

$$
(f(x)-f(y)) \delta^{\prime}(x-y)=-f^{\prime}(x) \delta(x-y)
$$

that Eq. (3), Eq. (4) and the relation

$$
\begin{aligned}
& \{D(x, \lambda), D(y, \mu)\}=-[r(x, \lambda, \mu) \delta(x-y), D(x, \lambda) \otimes 1+1 \otimes D(y, \mu)] \\
& +[s(x, \lambda, \mu) \delta(x-y), D(x, \lambda) \otimes 1-1 \otimes D(y, \mu)]
\end{aligned}
$$

are mutually equivalent.

Besides the $L$-matrix $L(x, \lambda)$ and the Lax operator $D(x, \lambda)$, another object of central importance in the theory of integrable systems is the transition matrix $T(x, y, \lambda)$, which is simply the corresponding parallel transport operator at fixed time,

$$
T(x, y, \lambda)=P \exp \int_{y}^{x} d z L(z, \lambda) .
$$

As argued, e.g., in [14], Eq: (3) or (4) implies the following basic Poisson brackets between the transition matrices:

$$
\begin{aligned}
&\{T(x, y, \lambda) \otimes T(u, v, \mu)\} \\
&=+\varepsilon(x-y) \chi(z ; x, y) \\
& \times\left.(T(x, z, \lambda) \otimes T(u, z, \mu))(r+s)(z, \lambda, \mu)(T(z, y, \lambda) \otimes T(z, v, \mu))\right|_{z=v} ^{z=u} \\
&+\varepsilon(u-v) \chi(z ; u, v) \\
& \times\left.(T(x, z, \lambda) \otimes T(u, z, \mu))(r-s)(z, \lambda, \mu)(T(z, y, \lambda) \otimes T(z, v, \mu))\right|_{z=y} ^{z=x} .
\end{aligned}
$$

Here $\varepsilon$ is the usual sign function,

$$
\varepsilon(x-y)=\left\{\begin{array}{rll}
+1 & \text { for } & x>y \\
0 & \text { for } & x=y \\
-1 & \text { for } & x<y
\end{array}\right.
$$

and $\chi(\cdot ; x, y)$ is the characteristic function of the interval between $x$ and $y$. These Poisson brackets are well-defined when considered as distributions in the respective variables and are continuous functions except at those points where two of their arguments $x, y, u, v$ coincide: there, they exhibit finite jumps proportional to $s$. Due to these discontinuities, the definition of Poisson brackets between transition matrices for coinciding or adjacent intervals, and in particular between monodromy matrices, is not completely straightforward (as would be the case for ultralocal models) but requires some kind of regularization. As a result, there arises the problem of finding a regularization scheme such that the fundamental algebraic properties of Poisson brackets, viz. the derivation rule and the Jacobi identity, remain valid for their regularized counterparts. A priori, it is not even clear whether such a regularization scheme exists at all, and indeed it has been suggested in the earlier literature that this is not the case [4]. Later, a prescription which amounts to a "total symmetrization over all possibly boundary values" was found to meet these requirements [14], but it is a multi-step regularization in the sense that regularization of multiple Poisson brackets with a given number of factors cannot be reduced to repeated regularization of multiple Poisson brackets with a smaller number of factors.

We conclude this introduction with a brief description of the contents of this paper. 
In Sect. 2 we briefly review the current algebra of integrable non-linear sigma models which has been derived in a previous paper [8] and further analysed in [11]. There it has been shown that the algebra closes provided that, in addition to the components $j_{\mu}$ of the Noether current, a new composite field $j$ is introduced.

In Sect. 3, we use this result to prove that the Poisson brackets between the $L$ matrices are indeed of the form (3), with matrices $r$ and $s$ which we determine explicitly as functions of the new field $j$. We also show how the Poisson brackets (9) for the transition matrices can be derived from the Poisson brackets (3) or (4) for the $L$-matrices, and we comment on the possibility of defining regularized Poisson brackets between transition matrices for coinciding or adjacent intervals, and in particular between monodromy matrices, by interpreting (9) as an equation for functions rather than distributions, which requires assigning specific values to the characteristic function $\chi$ at its points of discontinuity.

In Sect. 4, we complete our analysis by computing the Poisson brackets of the $L$-matrices with the matrices $r$ and $s$, which are non-trivial because $r$ and $s$ are field dependent. As it turns out, the Poisson brackets can all be expressed with the help of a new matrix $c$ which is numerical, i.e., field independent. In fact, the Lax operators $D$ and an appropriate linear combination $d$ of $r$ and $s$ close to a "quadratic algebra," i.e., their Poisson brackets are linear combinations of terms which are at most quadratic in $D$ and $d$. Similarly, the transition matrices $T$ and the same linear combination $d$ of $r$ and $s$ close to an algebra - at least when all expressions are considered as distributions in the respective variables. Finally, we return to the regularization procedure already discussed in the previous section, which is based on interpreting the basic Poisson brackets between the T's and $d$ 's as equations for functions rather than distributions and assigning specific values to the characteristic functions $\chi$ appearing there at their points of discontinuity - values that are assumed to be field independent but are allowed to depend in an arbitrary way on the spectral parameters involved. We find that a one-step regularization, which reduces the computation of regularized multiple Poisson brackets to a repeated application of the formula for the regularized basic Poisson brackets and the derivation rule, fails to satisfy the Jacobi identity.

In an appendix, we give, following [1], an explicit proof of a theorem on the classification of a class of asymmetric solutions to the classical Yang-Baxter equation, related to involutive automorphisms of semisimple Lie algebras.

Finally, we would like to mention a recent mathematical preprint [3] where "sufficiently generic" harmonic maps from the two-torus into Riemannian symmetric spaces are classified. The $r$-matrix arising there is however field independent and seems to play a different role than the $r$-matrix here.

\section{Current Algebra for Integrable Non-Linear Sigma Models}

We begin by briefly reviewing the results on the current algebra structure of general non-linear sigma models derived in a previous paper [8]. For simplicity, we restrict ourselves to the class of integrable non-linear sigma models, which are precisely those defined on Riemannian symmetric spaces $M=G / H$, and we shall use the gauge dependent formulation developed in [5,7] where the basic field $\varphi$ with values in $M$ is (at least locally) represented in terms of a field $g$ with values in $G$, determined modulo a field $h$ with values in $H$ by the condition that $\varphi=g H$. Technically, we require that $M$ is the quotient space of some (connected) Lie group $G$, with Lie 
algebra g, modulo some compact subgroup $H \subset G$, with Lie algebra $\mathbf{h} \subset \mathbf{g}$, and that there exists an $\operatorname{Ad}(H)$-invariant subspace $\mathbf{m}$ of $\mathbf{g}$, with commutation relations

$$
[\mathbf{h}, \mathbf{h}] \subset \mathbf{h}, \quad[\mathbf{h}, \mathbf{m}] \subset \mathbf{m}, \quad[\mathbf{m}, \mathbf{m}] \subset \mathbf{h},
$$

such that $\mathbf{g}$ is the (vector space) direct sum of $\mathbf{h}$ and $\mathbf{m}$ :

$$
\mathbf{g}=\mathbf{h} \oplus \mathbf{m} .
$$

The corresponding projections from $\mathbf{g}$ onto $\mathbf{h}$ along $\mathbf{m}$ and from $\mathbf{g}$ onto $\mathbf{m}$ along $\mathbf{h}$ will be denoted by $\pi_{\mathbf{h}}$ and $\pi_{\mathbf{m}}$, respectively. Moreover, we suppose that the $\operatorname{Ad}(H)$-invariant positive definite inner product $(\cdot, \cdot)$ on $\mathbf{m}$, corresponding to the given $G$-invariant Riemannian metric on $M$, is induced from an $\operatorname{Ad}(G)$-invariant non-degenerate inner product $(\cdot, \cdot)$ on $\mathbf{g}$, corresponding to a $G$-biinvariant pseudoRiemannian metric on $G$, so that the direct decomposition (12) is orthogonal. (For symmetric spaces of the compact or non-compact type, this hypothesis involves no loss of generality.) Then defining the covariant derivative $D_{\mu} g$ of $g$ to be

$$
D_{\mu} g=g \pi_{\mathbf{m}}\left(g^{-1} \partial_{\mu} g\right) \text {, }
$$

the action of the non-linear sigma model on $M=G / H$ can be written as

$$
S=\frac{1}{2} \int d^{2} x\left(\partial^{\mu} \varphi, \partial_{\mu} \varphi\right)=\frac{1}{2} \int d^{2} x\left(D^{\mu} g, D_{\mu} g\right) .
$$

The global $G$-invariance of this action leads to a Noether current $j_{\mu}$ taking values in $\mathbf{g :}$

$$
j_{\mu}=-D_{\mu} g g^{-1}
$$

As usual, the equations of motion imply (and in the models considered here are in fact equivalent to) current conservation

$$
\partial^{\mu} j_{\mu}=0
$$

In addition, the commutation relations (11) guarantee that $2 j_{\mu}$ also satisfies the zero curvature condition, i.e.,

$$
\partial_{\mu} j_{\nu}-\partial_{\nu} j_{\mu}+2\left[j_{\mu}, j_{\nu}\right]=0 \text {. }
$$

The other composite field which will be of central importance for all that follows is the scalar field $j$ taking values in the space $L(\mathbf{g}) \cong \mathbf{g} \otimes \mathbf{g}^{*}$ of linear transformations on $\mathbf{g}$ defined as

$$
j=\operatorname{Ad}(g) \pi_{\mathbf{m}} \operatorname{Ad}(g)^{-1} .
$$

It should be noted that the fields $j_{\mu}$ and $j$ are not independent. Thus for example, we have the algebraic identity

$$
\operatorname{ad}\left(j_{\mu}\right)=\left[j, \operatorname{ad}\left(j_{\mu}\right)\right]_{+}=j \operatorname{ad}\left(j_{\mu}\right)+\operatorname{ad}\left(j_{\mu}\right) j
$$

as well as an identity expressing the derivatives of $j$ in terms of $j$ and $j_{\mu}$ :

$$
\partial_{\mu} j=\left[j, \operatorname{ad}\left(j_{\mu}\right)\right]=j \operatorname{ad}\left(j_{\mu}\right)-\operatorname{ad}\left(j_{\mu}\right) j .
$$

The main motivation for introducing the field $j$ is that it is precisely the additional ingredient needed to write down the current algebra in closed form. To do so, it is convenient to introduce a basis $\left(T_{a}\right)$ of $\mathbf{g}$, with structure constants $f_{a b}^{c}$ defined by 
$\left[T_{a}, T_{b}\right]=f_{a b}^{c} T_{c}$ and metric coefficients $\eta_{a b}$ defined by $\left(T_{a}, T_{b}\right)=\eta_{a b}$, together with the corresponding dual basis $\left(T^{a}\right)$ of $\mathbf{g}^{*}$, and to expand $j_{\mu}$ and $j$ into components: ${ }^{3}$

$$
j_{\mu}=j_{\mu, a} T^{a}, \quad j=j_{a b} T^{a} \otimes T^{b} .
$$

With this notation, the current algebra (at fixed time $t$ ) takes the form [8]

$$
\begin{aligned}
& \left\{j_{0, a}(x), j_{0, b}(y)\right\}=-f_{a b}^{c} j_{0, c}(x) \delta(x-y), \\
& \left\{j_{0, a}(x), j_{1, b}(y)\right\}=-f_{a b}^{c} j_{1, c}(x) \delta(x-y)+j_{a b}(y) \delta^{\prime}(x-y), \\
& \left\{j_{1, a}(x), j_{1, b}(y)\right\}=0 \\
& \left\{j_{0, a}(x), j_{b c}(y)\right\}=-\left(f_{a b}^{d} j_{c d}(x)+f_{a c}^{d} j_{b d}(x)\right) \delta(x-y), \\
& \left\{j_{1, a}(x), j_{b c}(y)\right\}=0 \\
& \left\{j_{a b}(x), j_{c d}(y)\right\}=0 .
\end{aligned}
$$

\section{Fundamental Poisson Brackets}

Our goal in this section is to compute, for the models under consideration, Poisson brackets between various quantities which play a central role in the theory of twodimensional integrable field theories, such as the $L$-matrix $L(x, \lambda)$ or the transition matrix $T(x, y, \lambda)$ and the monodromy matrix $T(\lambda)$. Our starting point will of course be the current algebra (22)-(27), which governs the canonical structure of these models.

According to the commonly accepted point of view (see, e.g., [6]), integrability of a classical two-dimensional field theory is expressed through the possibility to rewrite its equations of motion as a zero curvature condition

$$
\partial_{\mu} L_{\nu}-\partial_{\nu} L_{\mu}+\left[L_{\mu}, L_{\nu}\right]=0,
$$

or equivalently, as the condition of commutativity

$$
\left[D_{\mu}, D_{\nu}\right]=0
$$

for the covariant derivatives

$$
D_{\mu}=\partial_{\mu}+L_{\mu} .
$$

This is the compatibility condition for the linear system (Lax pair)

$$
\partial_{\mu} U=U L_{\mu} .
$$

Here $U$ and $L_{\mu}$ are functions on two-dimensional space-time taking values in an appropriate Lie group $G$ and in the corresponding Lie algebra $\mathbf{g}$, respectively, and depending on an additional spectral parameter $\lambda$. In the present case, $L_{\mu}$ is simply

\footnotetext{
3 According to the original definition, valid for arbitrary Riemannian manifolds, $j_{\mu}$ should be considered as taking its values in the dual space $\mathbf{g}^{*}$ of $\mathbf{g}$ while $j$ should be considered as taking its values in the symmetric tensor product of $\mathbf{g}^{*}$ with itself. The point of view taken above only emerges after identifying $\mathbf{g}$ with $\mathbf{g}^{*}$ by means of the invariant scalar product $(\cdot, \cdot)$ on $\mathbf{g}$, or in component language, by using the corresponding metric coefficients to pull up and down Lie algebra indices
} 
an appropriate $\lambda$-dependent linear combination of the current $j_{\mu}$ and its (Hodge) dual $\varepsilon_{\mu \nu} j^{\nu}$, namely ${ }^{4}$

$$
L_{\mu}=\frac{2}{1-\lambda^{2}}\left(j_{\mu}+\lambda \varepsilon_{\mu \nu} j^{\nu}\right) .
$$

Indeed, with this choice, the $\lambda$-dependent zero curvature condition (28) is equivalent to both current conservation (16) and the $\lambda$-independent zero curvature condition (17).

Next, recall that the $L$-matrix $L$ is defined to be the spatial component of the flat vector potential $L_{\mu}$,

$$
L(x, \lambda)=\frac{2}{1-\lambda^{2}}\left(j_{1}(x)+\lambda j_{0}(x)\right),
$$

while the transition matrix $T(x, y, \lambda)$ is simply the parallel transport operator from $y$ to $x$ (at fixed time $t$ ) associated with the flat vector potential $L_{\mu}$, so in terms of solutions $U$ of the linear system (31), it is given by

$$
T(x, y, \lambda)=U(x, \lambda)^{-1} U(y, \lambda) .
$$

Therefore it obeys the differential equations

$$
\frac{\partial}{\partial x} T(x, y, \lambda)=-L(x, \lambda) T(x, y, \lambda)
$$

and

$$
\frac{\partial}{\partial y} T(x, y, \lambda)=+T(x, y, \lambda) L(y, \lambda)
$$

with initial condition

$$
\left.T(x, y, \lambda)\right|_{x=y}=1
$$

as well as the composition rule

$$
T(x, y, \lambda) T(y, z, \lambda)=T(x, z, \lambda),
$$

which, together with (37), leads to the inversion formula

$$
T(x, y, \lambda)^{-1}=T(y, x, \lambda) .
$$

Moreover, under standard boundary conditions on the fields at spatial infinity $(\varphi$ approaches a given point in $M$ and all of its derivatives vanish sufficiently rapidly), the limit

$$
T(\lambda)=\lim _{\substack{x \rightarrow+\infty \\ y \rightarrow-\infty}} T(x, y, \lambda)
$$

exists: this is the monodromy matrix.

Turning to the actual calculation of Poisson brackets, we first expand $L$ into components

$$
L=L_{a} T^{a}
$$

\footnotetext{
4 Our convention for the spectral parameter follows that of [6, p. 312] and [12]; it is related to the spectral parameter used in [4] and [14] by the inversion $\lambda \rightarrow \lambda^{-1}$ and to the spectral parameter $\gamma$ employed in [5,7] by the Möbius transformation $\lambda \rightarrow \gamma=(\lambda-1) /(\lambda+1)$. Our convention for the $\varepsilon$-tensor is $\varepsilon_{01}=-1$
} 
cf. (21). In these terms, the result follows directly from combining the current algebra (22)-(27) with the definition (33) of $L$ :

$$
\begin{aligned}
\left\{L_{a}(x, \lambda), L_{b}(y, \mu)\right\}= & \frac{2 f_{a b}^{c}}{\lambda-\mu}\left(\frac{\mu^{2}}{1-\mu^{2}} L_{c}(x, \lambda)-\frac{\lambda^{2}}{1-\lambda^{2}} L_{c}(x, \mu)\right) \delta(x-y) \\
& +\frac{4}{\left(1-\lambda^{2}\right)\left(1-\mu^{2}\right)}\left(\mu j_{a b}(x)+\lambda j_{a b}(y)\right) \delta^{\prime}(x-y), \\
\left\{L_{a}(x, \lambda), j_{b c}(y)\right\}= & -\frac{2 \lambda}{1-\lambda^{2}}\left(f_{a b}^{d} j_{c d}(x)+f_{a c}^{d} j_{b d}(x)\right) \delta(x-y) . \\
\left.\left\{j_{a b} x\right), j_{c d}(y)\right\}= & 0 .
\end{aligned}
$$

The next step will be to rewrite the first of these equations in terms of the usual tensor notation [6] and to show that it can in fact be cast into the form (3) or (4) given by Maillet [14]. To do so, we embed $\mathbf{g}$ into its universal enveloping algebra $U(\mathbf{g})$ and consider the fields $L$ and $j$ as taking values in $U(\mathbf{g})$ and in $U(\mathbf{g}) \otimes U(\mathbf{g})$, respectively. Similarly, the Casimir tensor $C$, defined by

$$
C=\eta^{a b} T_{a} \otimes T_{b},
$$

is viewed as an element of $U(\mathbf{g}) \otimes U(\mathbf{g})$. Then we have, for example,

$$
\left[C, T_{c} \otimes 1\right]=+f_{c}^{a b} T_{a} \otimes T_{b}, \quad\left[C, 1 \otimes T_{c}\right]=-f_{c}^{a b} T_{a} \otimes T_{b},
$$

while

$$
\left[j, T_{c} \otimes 1\right]=-f_{c d}^{a} j^{b d} T_{a} \otimes T_{b}, \quad\left[j, 1 \otimes T_{c}\right]=-f_{c d}^{b} j^{a d} T_{a} \otimes T_{b},
$$

so that the identities (19) and (20) become

$$
\left[C, j_{\mu} \otimes 1\right]=-\left[C, 1 \otimes j_{\mu}\right]=\left[j, j_{\mu} \otimes 1-1 \otimes j_{\mu}\right],
$$

and

$$
\partial_{\mu} j=\left[j, j_{\mu} \otimes 1+1 \otimes j_{\mu}\right]
$$

respectively; they can be combined into one identity involving $L$ instead of the $j_{\mu}$ :

$$
\begin{aligned}
& \frac{2(\lambda-\mu)}{\left(1-\lambda^{2}\right)\left(1-\mu^{2}\right)} j^{\prime}(x) \\
& \quad=\left[C-2 j(x), \frac{\mu}{1-\mu^{2}} L(x, \lambda) \otimes 1-\frac{\lambda}{1-\lambda^{2}} 1 \otimes L(x, \mu)\right] .
\end{aligned}
$$

Therefore, we can rewrite the Poisson bracket relation (42) in the form

$$
\begin{aligned}
\{L(x, \lambda) \stackrel{\otimes}{,} L(y, \mu)\}= & {\left[\frac{2 C}{\lambda-\mu}, \frac{\mu^{2}}{1-\mu^{2}} L(x, \lambda) \otimes 1+\frac{\lambda^{2}}{1-\lambda^{2}} 1 \otimes L(x, \mu)\right] \delta(x-y) } \\
& -\frac{2(\lambda-\mu)}{\left(1-\lambda^{2}\right)\left(1-\mu^{2}\right)}(j(x)-j(y)) \delta^{\prime}(x-y) \\
& +\frac{2(\lambda+\mu)}{\left(1-\lambda^{2}\right)\left(1-\mu^{2}\right)}(j(x)+j(y)) \delta^{\prime}(x-y) .
\end{aligned}
$$


Using the identities (6), (50) and collecting the terms gives

$$
\begin{aligned}
& \{L(x, \lambda) \stackrel{\otimes}{,} L(y, \mu)\} \\
& =\left[\frac{\lambda+\mu}{\lambda-\mu} C, \frac{\mu}{1-\mu^{2}} L(x, \lambda) \otimes 1+\frac{\lambda}{1-\lambda^{2}} 1 \otimes L(x, \mu)\right] \delta(x-y) \\
& \quad-\left[2 j(x), \frac{\mu}{1-\mu^{2}} L(x, \lambda) \otimes 1-\frac{\lambda}{1-\lambda^{2}} 1 \otimes L(x, \mu)\right] \delta(x-y) \\
& \quad+\frac{2(\lambda+\mu)}{\left(1-\lambda^{2}\right)\left(1-\mu^{2}\right)}(j(x)+j(y)) \delta^{\prime}(x-y) .
\end{aligned}
$$

Comparing this with the rhs of Eq. (4) suggests the following Ansatz for $r$ and $s$ :

$$
r(z, \lambda, \mu)=a(\lambda, \mu) C+b(\lambda, \mu) j(z),
$$

with coefficients $a(\lambda, \mu)$ and $b(\lambda, \mu)$ to be determined, and

$$
s(z, \lambda, \mu)=-\frac{2(\lambda+\mu)}{\left(1-\lambda^{2}\right)\left(1-\mu^{2}\right)} j(z) .
$$

Then applying the identity (50) again, Eq. (4) becomes, after a short calculation

$$
\begin{aligned}
\{L(x, \lambda) & \stackrel{\otimes}{,} L(y, \mu)\} \\
= & -\left(a(\lambda, \mu)-b(\lambda, \mu) \frac{\mu\left(1-\lambda^{2}\right)}{2(\lambda-\mu)}\right)[C, L(x, \lambda) \otimes 1] \delta(x-y) \\
& -\left(a(\lambda, \mu)+b(\lambda, \mu) \frac{\lambda\left(1-\mu^{2}\right)}{2(\lambda-\mu)}\right)[C, 1 \otimes L(x, \mu)] \delta(x-y) \\
& -\left(b(\lambda, \mu)\left(1+\frac{\mu\left(1-\lambda^{2}\right)}{\lambda-\mu}\right)+\frac{2(\lambda+\mu)}{\left(1-\lambda^{2}\right)\left(1-\mu^{2}\right)}\right)[j(x), L(x, \lambda) \otimes 1] \delta(x-y) \\
& -\left(b(\lambda, \mu)\left(1-\frac{\lambda\left(1-\mu^{2}\right)}{\lambda-\mu}\right)-\frac{2(\lambda+\mu)}{\left(1-\lambda^{2}\right)\left(1-\mu^{2}\right)}\right)[j(x), 1 \otimes L(x, \mu)] \delta(x-y) \\
& +\frac{2(\lambda+\mu)}{\left(1-\lambda^{2}\right)\left(1-\mu^{2}\right)}(j(x)+j(y)) \delta^{\prime}(x-y) .
\end{aligned}
$$

Comparing once more, we arrive at the following solution:

$$
a(\lambda, \mu)=-\frac{2 \lambda \mu}{(1-\lambda \mu)(\lambda-\mu)}, \quad b(\lambda, \mu)=-\frac{2(1+\lambda \mu)(\lambda-\mu)}{(1-\lambda \mu)\left(1-\lambda^{2}\right)\left(1-\mu^{2}\right)} .
$$

Thus we infer that the Poisson brackets between the $L$-matrices can indeed be brought into the form (3), (4) if we choose $r$ and $s$ to be given by

$$
\begin{gathered}
r(z, \lambda, \mu)=-\frac{2 \lambda \mu}{(1-\lambda \mu)(\lambda-\mu)} C-\frac{2(1+\lambda \mu)(\lambda-\mu)}{(1-\lambda \mu)\left(1-\lambda^{2}\right)\left(1-\mu^{2}\right)} j(z), \\
s(z, \lambda, \mu)=-\frac{2(\lambda+\mu)}{\left(1-\lambda^{2}\right)\left(1-\mu^{2}\right)} j(z) .
\end{gathered}
$$

For later use, we also define $d$ to be the difference $s-r$,

$$
d(z, \lambda, \mu)=(s-r)(z, \lambda, \mu)=\frac{2 \lambda \mu}{1-\lambda \mu}\left(\frac{C}{\lambda-\mu}-\frac{2 j(z)}{\lambda\left(1-\mu^{2}\right)}\right),
$$


and set

$$
c(z, \lambda, \mu)=\frac{2 \lambda}{1-\lambda^{2}} C .
$$

Moreover, the functions $r, s, d$, and $c$ of one spatial argument are extended to distributions in two spatial arguments, again denoted by $r, s, d$, and $c$, respectively, by multiplying them with a delta function in the difference of the arguments:

$$
\begin{array}{ll}
r(x, \lambda ; y, \mu)=r(x, \lambda, \mu) \delta(x-y), & s(x, \lambda ; y, \mu)=s(x, \lambda, \mu) \delta(x-y), \\
d(x, \lambda ; y, \mu)=d(x, \lambda, \mu) \delta(x-y), & c(x, \lambda ; y, \mu)=c(x, \lambda, \mu) \delta(x-y) .
\end{array}
$$

We conclude this section with some remarks on the calculation of Poisson brackets for transition matrices and monodromy matrices. Our discussion essentially follows [14], to which we refer for further reading.

The Poisson brackets between transition matrices can be obtained from those between $L$-matrices by making use of a well-known formula for the variation of the $T$ 's induced by a variation of the $L$ 's (cf. [6, pp. 191/192]): ${ }^{5}$

$$
\delta T(x, y, \lambda)=\int d z \varepsilon(x-y) \chi(z ; x, y) T(x, z, \lambda) \delta L(z, \lambda) T(z, y, \lambda) .
$$

Here $\varepsilon$ is the usual sign function [cf. (10)], while $\chi(\cdot ; x, y)$ is the characteristic function of the interval between $x$ and $y:{ }^{6}$

$$
\chi(z ; x, y)= \begin{cases}\alpha & \text { for } z=\min \{x, y\} \\ 1 & \text { for } \min \{x, y\}<z<\max \{x, y\} \\ \beta & \text { for } z=\max \{x, y\} \\ 0 & \text { otherwise }\end{cases}
$$

Note that

$$
\begin{aligned}
\varepsilon(x-y) \frac{\partial}{\partial z} \chi(z ; x, y) & =\varepsilon(x-y)(\delta(z-\min \{x, y\})-\delta(z-\max \{x, y\})) \\
& =\delta(z-y)-\delta(z-x)
\end{aligned}
$$

Now by the chain rule (applied to the functional derivatives involved in the definition of the Poisson bracket), we have (cf. [6, p. 192])

$$
\begin{aligned}
& \{T(x, y, \lambda) \stackrel{\otimes}{,} T(u, v, \mu)\} \\
& =\int d z d w \varepsilon(x-y) \varepsilon(u-v) \chi(z ; x, y) \chi(w ; u, v) \\
& \quad \times(T(x, z, \lambda) \otimes T(u, w, \mu))\{L(z, \lambda) \stackrel{\otimes}{,} L(w, \mu)\}(T(z, y, \lambda) \otimes T(w, v, \mu)) .
\end{aligned}
$$

Similarly,

$$
\begin{aligned}
\{T(x, y, \lambda) \stackrel{\otimes}{,} L(z, \mu)\}= & \int d z^{\prime} \varepsilon(x-y) \chi\left(z^{\prime} ; x, y\right)\left(T\left(x, z^{\prime}, \lambda\right) \otimes 1\right) \\
& \times\left\{L\left(z^{\prime}, \lambda\right) \stackrel{\otimes}{,} L(z, \mu)\right\}\left(T\left(z^{\prime}, y, \lambda\right) \otimes 1\right) .
\end{aligned}
$$

\footnotetext{
5 In the proof, the case $x<y$ can be reduced to the case $x>y$ discussed in [6] by means of the inversion formula (39), and of course (56) is trivially satisfied if $x=y$

6 The values $\alpha$ and $\beta$ of $\chi(z ; x, y)$ at the points of discontinuity, $z=x$ and $z=y$, are of course irrelevant at this stage: they do however play a role in the regularization procedure needed to define Poisson brackets of transition matrices at coinciding points, as will be explained in more detail below
} 
Inserting the Poisson bracket relation (4), transforming the $\delta^{\prime}$-term into a $\delta$-term by partial integration and using the differential equations (35), (36) together with Eq. (58), we can perform the integral over $z^{\prime}$, with the result

$$
\begin{aligned}
&\{T(x, y, \lambda) \stackrel{\otimes}{,} L(z, \mu)\} \\
&=-2(\delta(z-x)-\delta(z-y))(T(x, z, \lambda) \otimes 1) s(z, \lambda, \mu)(T(z, y, \lambda) \otimes 1) \\
&+\varepsilon(x-y) \chi(z ; x, y)(T(x, z, \lambda) \otimes 1) \\
& \times\left\{(r+s)^{\prime}(z, \lambda, \mu)-[(r+s)(z, \lambda, \mu), L(z, \lambda) \otimes 1+1 \otimes L(z, \mu)]\right\} \\
& \times(T(z, y, \lambda) \otimes 1) .
\end{aligned}
$$

Finally,

$$
\begin{aligned}
\{T(x, y, \lambda) \stackrel{\otimes}{,} T(u, v, \mu)\}= & \int d z \varepsilon(u-v) \chi(z ; u, v)(1 \otimes T(u, z, \mu)) \\
& \times\{T(x, y, \lambda) \stackrel{\otimes}{,} L(z, \mu)\}(1 \otimes T(z, v, \mu)) .
\end{aligned}
$$

Inserting the Poisson bracket relation (61) just obtained and using the differential equations (35), (36), we arrive at

$$
\begin{aligned}
\{T(x, y, \lambda) & \stackrel{\otimes}{,} T(u, v, \mu)\} \\
= & -2 \varepsilon(u-v) \chi(z ; u, v) \\
& \times\left.(T(x, z, \lambda) \otimes T(u, z, \mu)) s(z, \lambda, \mu)(T(z, y, \lambda) \otimes T(z, v, \mu))\right|_{z=y} ^{z=x} \\
& +\int d z \varepsilon(x-y) \varepsilon(u-v) \chi(z ; x, y) \chi(z ; u, v) \\
& \times \frac{\partial}{\partial z}((T(x, z, \lambda) \otimes T(u, z, \mu))(r+s)(z, \lambda, \mu)(T(z, y, \lambda) \otimes T(z, v, \mu)))
\end{aligned}
$$

where the integral over $z$ can be performed using Eq. (58): the result is Eq. (9).

The Poisson brackets between monodromy matrices are usually derived by specializing the Poisson brackets between transition matrices to the case of coinciding intervals and then sending the boundaries of the interval to infinity - a procedure which is known to work well in the ultralocal case. In general, however, expressions such as $\{L(x, \lambda) \stackrel{\otimes}{,} L(y, \mu)\},\{T(x, y, \lambda) \stackrel{\otimes}{,} L(z, \mu)\}$ and $\{T(x, y, \lambda) \stackrel{\otimes}{,} T(u, v, \mu)\}$ are distributions in the respective variables, with singular support on the set of points where (at least) two of their arguments coincide (a set which, geometrically, is a union of hyperplanes). Therefore, defining Poisson brackets between transition matrices for coinciding intervals or for adjacent intervals will normally require some kind of regularization. Now in the ultralocal case, $\{L(x, \lambda) \stackrel{\otimes}{,} L(y, \mu)\}$ has only a $\delta$-singularity at $y=x$ and hence ${ }^{7}\{T(x, y, \lambda) \stackrel{\otimes}{,} T(u, v, \mu)\}$ is a continuous function everywhere, so that no regularization is needed. But in the situation under consideration here, $\{L(x, \lambda) \stackrel{\otimes}{,} L(y, \mu)\}$ has a $\delta^{\prime}$-singularity at $y=x$ and hence ${ }^{7}\{T(x, y, \lambda) \stackrel{\otimes}{,} T(u, v, \mu)\}$ is discontinuous, with a finite jump proportional to the relevant value of $s$, whenever $u=x$ or $u=y$ or $v=x$ or $v=y$. The simplest possibility to regularize this expression at the points of discontinuity is to just take the average over all possible boundary values: this "total symmetrization rule" is the prescription employed by Maillet [14]. It leads to

$$
\begin{aligned}
\{T(x, y, \lambda) \stackrel{\otimes}{,} T(x, y, \mu)\}= & \varepsilon(x-y)(r(x, \lambda, \mu)(T(x, y, \lambda) \otimes T(x, y, \mu)) \\
& -(T(x, y, \lambda) \otimes T(x, y, \mu)) r(y, \lambda, \mu)),
\end{aligned}
$$

\footnotetext{
7 Roughly speaking, the Poisson brackets between the $T$ 's are obtained from those between the $L$ 's by a twofold integration: this is reflected in the nature of the singularities
} 
and to

$$
\{T(x, y, \lambda) \stackrel{\otimes}{,} T(y, z, \mu)\}=\varepsilon(x-z)(T(x, y, \lambda) \otimes 1) s(y, \lambda, \mu)(1 \otimes T(y, z, \mu)) .
$$

Obviously, Eq. (64) implies

$$
\{T(\lambda) \stackrel{\otimes}{,} T(\mu)\}=r_{+}(\lambda, \mu)(T(\lambda) \otimes T(\mu))-(T(\lambda) \otimes T(\mu)) r_{-}(\lambda, \mu)
$$

with

$$
r_{ \pm}(\lambda, \mu)=\lim _{z \rightarrow \pm \infty} r(z, \lambda, \mu)
$$

A slightly more general regularization procedure would be to consider (9) not as an equation between distributions but rather as one between functions, i.e., to postulate its validity even at the points of discontinuity. In this way, the (so far irrelevant) parameters $\alpha$ and $\beta$ introduced in Eq. (57) acquire a definite meaning. They are however not independent, because requiring the derivation rule

$$
\begin{aligned}
& \{T(x, y, \lambda) T(y, z, \lambda) \stackrel{\otimes}{,} A\} \\
& \quad=\{T(x, y, \lambda) \stackrel{\otimes}{,} A\}(T(y, z, \lambda) \otimes 1)+(T(x, y, \lambda) \otimes 1)\{T(y, z, \lambda) \stackrel{\otimes}{,} A\}
\end{aligned}
$$

to be valid, e.g., with $A=L(w, \mu)$, not only in the sense of distributions but also in the sense of functions, i.e., even at $w=y$, forces them to satisfy the constraint $\alpha+\beta=1$. In particular, this constraint holds for the "total symmetrization rule" referred to above, which corresponds to the simplest choice $\alpha=\frac{1}{2}$ and $\beta=\frac{1}{2}$, giving

$$
\chi(z ; x, y)=\theta(z-\min \{x, y\}) \theta(\max \{x, y\}-z)
$$

with $\theta(0)=\frac{1}{2}$. More general prescriptions will be discussed in the next section.

The main difficulty with the above derivation of a priori ill-defined Poisson brackets is the one associated with any regularization procedure: one cannot be sure that algebraic relations between the unregularized quantities remain valid for the regularized expressions. This is a well-known and fundamental problem in quantum field theory, being the origin, e.g., for the occurrence of anomalies. In the present case, the algebraic relation in question is the Jacobi identity. In particular, we have seen that the monodromy matrices are subject to the standard Poisson bracket relations (66) of the $r$-matrix approach [6], but with asymptotic $r$-matrices $r_{+}$and $r_{-}$which - according to the classification theorem given in the appendix - do not satisfy the classical Yang-Baxter equation, i.e., the relation normally imposed in order to guarantee that the Jacobi identity holds. This apparent paradox can be resolved by remembering that the lhs of Eq. (66) is a regularized Poisson bracket and noting that of course the corresponding Jacobi identity must be regularized as well. In fact, the regularized Jacobi identity will involve regularized double Poisson brackets which are not identical with the double Poisson brackets one would normally obtain from a naive twofold application of Eq. (66), and hence its validity will be governed by an equation which is not identical with the classical Yang-Baxter equation. To derive it, one must go back to the definitions and must compute double Poisson brackets between transition matrices, which necessarily involve simple Poisson brackets between transition matrices and the matrices $r \pm s$ : their calculation will be one of our goals in the next section.

Before proceeding, we would like to point out that an alternative and more direct method for deriving Poisson brackets between transition matrices at coinciding points, without recourse to an explicit regularization, has been proposed in the recent literature 
[9]. It is based on expressing the $L$-matrix as a gauge transform of some other matrix in such a way that both this other matrix and the gauge transformation itself satisfy Poisson bracket relations of ultralocal type. This approach does work for the principal chiral models, in which the coefficient of the classical Schwinger term is both field independent and central - at least as long as one considers only left currents or only right currents. But it is not clear at all whether the technique can be extended to the situation of interest here, which is substantially more general. This is certainly an interesting question but also quite a difficult one, which warrants a separate investigation. For the time being, we have preferred to stick to the more traditional method.

\section{The Lie-Poisson Structure}

In the preceding section, we have rewritten the fundamental Poisson brackets between $L$-matrices in tensor notation and have discussed some consequences that can be drawn, such as the calculation of the fundamental Poisson brackets between transition matrices and monodromy matrices. Our goal now is to exhibit the full Lie-Poisson structure of the theory - which in terms of components is contained in Eqs. (42)-(44) - and to discuss the algebraic constraints resulting from the Jacobi identity for Poisson brackets. This requires, first of all, a slight modification of the tensor notation used previously, adapted to deal not only with the tensor product $U(\mathbf{g}) \otimes U(\mathbf{g})$ of $U(\mathbf{g})$ with itself but with its tensor powers $U(\mathbf{g})^{\otimes n}$ of arbitrary order and with the various ways in which these can be embedded into each other. For example, note that $U(\mathbf{g})$ and $U(\mathbf{g}) \otimes U(\mathbf{g})$ can be embedded into $U(\mathbf{g})^{\otimes n}$ according to

$$
U(\mathbf{g}) \rightarrow U(\mathbf{g})^{\otimes n}, \quad u \mapsto u_{k}=1 \otimes \ldots \otimes u \otimes \ldots \otimes 1
$$

(with $u$ appearing in the $k^{\text {th }}$ place, $1 \leq k \leq n$ ) and to

$$
U(\mathbf{g}) \otimes U(\mathbf{g}) \rightarrow U(\mathbf{g})^{\otimes n}, \quad u \otimes v \mapsto(u \otimes v)_{k l}=1 \otimes \ldots \otimes u \otimes \ldots \otimes v \otimes \ldots \otimes 1
$$

(with $u$ appearing in the $k^{\text {th }}$ place and $v$ appearing in the $l^{\text {th }}$ place, $1 \leq k, l \leq n$ ), respectively. Moreover, we shall find it convenient to introduce, besides the composite field $j$ used so far, a new composite field $\sigma$, the two being related by $\sigma=1-2 j$ when both are considered as taking values in the space $L(\mathbf{g}) \cong \mathbf{g} \otimes \mathbf{g}^{*}$ of linear transformations on $\mathbf{g}$ (with 1 denoting the identity on $\mathbf{g}$ ), or equivalently, by $\sigma=C-2 j$ when both are considered as taking values in $U(\mathbf{g}) \otimes U(\mathbf{g})$. Then, for $k, l, m$ all distinct, we have

$$
\begin{gathered}
{\left[C_{k l}, C_{k m}\right]=f^{a b c}\left(T_{a}\right)_{k}\left(T_{b}\right)_{l}\left(T_{c}\right)_{m},} \\
{\left[C_{k l}, \sigma_{k m}\right]=f_{d}^{a b} \sigma^{d c}\left(T_{a}\right)_{k}\left(T_{b}\right)_{l}\left(T_{c}\right)_{m},} \\
{\left[\sigma_{k l}, \sigma_{k m}\right]=f_{d e}^{a} \sigma^{d b} \sigma^{e c}\left(T_{a}\right)_{k}\left(T_{b}\right)_{l}\left(T_{c}\right)_{m}=f_{d}^{b c} \sigma^{d a}\left(T_{a}\right)_{k}\left(T_{b}\right)_{l}\left(T_{c}\right)_{m},}
\end{gathered}
$$

where the second equality in the last equation is based on the fact that we are dealing with a symmetric space, so that $\sigma$ is a (field dependent) involutive automorphism of g. In particular, we have, for $k, l, m, n$ all distinct, the following algebraic identities:

$$
\begin{gathered}
{\left[C_{k l}, C_{k m}\right]=-\left[C_{k l}, C_{l m}\right],} \\
{\left[C_{k l}, \sigma_{k m}\right]=-\left[C_{k l}, \sigma_{l m}\right],} \\
{\left[\sigma_{k l}, \sigma_{k m}\right]=-\left[\sigma_{k l}, C_{l m}\right],} \\
{\left[C_{k l}, C_{m n}\right]=\left[C_{k l}, \sigma_{m n}\right]=\left[\sigma_{k l}, \sigma_{m n}\right]=0 .}
\end{gathered}
$$


In this notation, the Poisson brackets for the $L$ 's and $j$ 's given by Eqs. (42)-(44) read

$$
\begin{aligned}
& \left\{L_{k}\left(x_{k}, \lambda_{k}\right), L_{l}\left(x_{l}, \lambda_{l}\right)\right\} \\
& =\left[\frac{2 C_{k l}}{\lambda_{k}-\lambda_{l}}, \frac{\lambda_{l}^{2}}{1-\lambda_{l}^{2}} L_{k}\left(x_{k}, \lambda_{k}\right)+\frac{\lambda_{k}^{2}}{1-\lambda_{k}^{2}} L_{l}\left(x_{l}, \lambda_{l}\right)\right] \delta\left(x_{k}-x_{l}\right) \\
& \quad+\frac{4}{\left(1-\lambda_{k}^{2}\right)\left(1-\lambda_{l}^{2}\right)}\left(\lambda_{l} j_{k l}\left(x_{k}\right)+\lambda_{k} j_{k l}\left(x_{l}\right)\right) \delta^{\prime}\left(x_{k}-x_{l}\right), \\
& \left\{L_{k}\left(x_{k}, \lambda_{k}\right), j_{l m}\left(x_{l}\right)\right\}=\frac{2 \lambda_{k}}{1-\lambda_{k}^{2}}\left[C_{k l}+C_{k m}, j_{l m}\left(x_{k}\right)\right] \delta\left(x_{k}-x_{l}\right), \\
& \left\{j_{k l}\left(x_{k}\right), j_{m n}\left(x_{m}\right)\right\}=0 .
\end{aligned}
$$

Observe that due to the identity (69), Eq. (74) continues to hold when $j$ is replaced by $\sigma$ or by any other linear combination of $C$ and $j$, such as $r, s$, or $d$. Therefore, the Poisson brackets for the $D$ 's and $d$ 's [cf. (5), (7), (53)-(55)] take the following, much more transparent form:

$$
\begin{aligned}
& \left\{D_{k}\left(x_{k}, \lambda_{k}\right), D_{l}\left(x_{l}, \lambda_{l}\right)\right\} \\
& \quad=\left[d_{k l}\left(x_{k}, \lambda_{k} ; x_{l}, \lambda_{l}\right), D_{k}\left(x_{k}, \lambda_{k}\right)\right]-\left[d_{l k}\left(x_{l}, \lambda_{l} ; x_{k}, \lambda_{k}\right), D_{l}\left(x_{l}, \lambda_{l}\right)\right], \\
& \left\{D_{k}\left(x_{k}, \lambda_{k}\right), d_{l m}\left(x_{l}, \lambda_{l} ; x_{m}, \lambda_{m}\right)\right\} \\
& \quad=\left[c_{k l}\left(x_{k}, \lambda_{k} ; x_{l}, \lambda_{l}\right)+c_{k m}\left(x_{k}, \lambda_{k} ; x_{m}, \lambda_{m}\right), d_{l m}\left(x_{l}, \lambda_{l} ; x_{m}, \lambda_{m}\right)\right] \\
& \quad\left\{d_{k l}\left(x_{k}, \lambda_{k} ; x_{l}, \lambda_{l}\right), d_{m n}\left(x_{m}, \lambda_{m} ; x_{n}, \lambda_{n}\right)\right\}=0,
\end{aligned}
$$

where, as elements of $U(\mathbf{g})^{\otimes n}, d_{l k}=d_{k l}$ and $c_{l k}=c_{k l}$, because the tensors $C$ and $j$ are symmetric. Obviously, these formulas can be further simplified by letting the indices $k, l, m$ refer not only to the position inside the tensor product but also to the spatial variable and to the spectral parameter, so $k, l$ and $m$ now stand for $\left(k, x_{k}, \lambda_{k}\right)$, $\left(l, x_{l}, \lambda_{l}\right)$ and $\left(m, x_{m}, \lambda_{m}\right)$, respectively. In these composite indices, $d$ and $c$ are of course no longer symmetric, $d_{l k} \neq d_{k l}, c_{l k} \neq c_{k l}$, but (76)-(78) reduce to

$$
\begin{aligned}
\left\{D_{k}, D_{l}\right\} & =\left[d_{k l}, D_{k}\right]-\left[d_{l k}, D_{l}\right], \\
\left\{D_{k}, d_{l m}\right\} & =\left[c_{k l}+c_{k m}, d_{l m}\right], \\
\left\{d_{k l}, d_{m n}\right\} & =0 .
\end{aligned}
$$

The first of these equations has appeared in the literature before [2], but the others seem to be new. Together, they show that the $D$ 's and $d$ 's generate an algebra which closes under Poisson brackets, because $c$ is a numerical (i.e., field independent) matrix [cf. (54)]. In the theory of non-ultralocal integrable models of the type considered here, this algebra plays a central role: it is the analogue of the classical YangBaxter algebra which is relevant to ultralocal integrable models such as, e.g., the non-linear Schrödinger equation [6]. But in order to give a mathematically more precise interpretation, one must make a clear-cut distinction between the abstract algebra and its concrete representations. For the classical Yang-Baxter algebras, this distinction is well understood: the structure of the abstract algebra is reflected in the $r$-matrix, which satisfies the classical Yang-Baxter equation (2), while the $L$ 's define a concrete representation of that algebra by functionals on the phase space of the theory, according to Eq. (1). Here, the structure of the abstract algebra is (at least partially) reflected in the $c$-matrix, while the $D$ 's and $d$ 's define a concrete representation of that 
algebra by functionals on the phase space of the theory, according to Eqs. (76)-(78) or (79)-(81). In particular, the $r$-matrix - which in this context is the antisymmetric part of the (field dependent) $d$-matrix - now has a different meaning.

The investigation of the mathematical structures that underlie this new algebra is still a completely open subject. The first step would be to identify its defining relations, i.e., the analogue of the Jacobi identity for Lie algebras or the classical Yang-Baxter equation for classical Yang-Baxter algebras: they should include a structure equation for the $c$-matrix which we suspect, once again, to be quadratic. Another problem would be to develop a representation theory for such algebras, and in particular, study their representations in Poisson algebras of function(al)s on phase spaces. All of this must be left to future work. Here, we just want to analyze the consequences of the fact that Eqs. (76)-(78) or (79)-(81) must be consistent with the Jacobi identity for Poisson brackets.

In order to do so, we first rewrite the definition (53) of $d$ in terms of $C$ and $\sigma$, rather than $C$ and $j$ :

$$
d(z, \lambda, \mu)=(s-r)(z, \lambda, \mu)=\frac{2 \mu}{1-\mu^{2}}\left(\frac{\mu C}{\lambda-\mu}+\frac{\sigma(z)}{1-\lambda \mu}\right) .
$$

Moreover, we shall find it convenient to use the following general notation. Given an arbitrary associative algebra with unit $\mathscr{A}$, we extend the tensor notation introduced above from $U(\mathbf{g})$ to $\mathscr{A}_{\mathrm{b}}$ and define, for $t \in \mathscr{b} \otimes \mathscr{A}_{\mathrm{s}}$ and $k, l, m$ all distinct,

$$
\mathrm{YB}(t)_{k l m}=\left[t_{k l}, t_{k m}\right]+\left[t_{k l}, t_{l m}\right]-\left[t_{k m}, t_{m l}\right] .
$$

Note the somewhat unusual position of the indices in the last term, which has however appeared in the literature before $[1,2,14]$. Of course, for antisymmetric $t$ (and with $\{k, l, m\}=\{1,2,3\})$, this expression reduces to the familiar lhs of the classical Yang-Baxter equation

$$
\mathrm{YB}(t)_{k l m}=0 \text {. }
$$

In the appendix, we compute $\mathrm{YB}(t)$ for $t$ an arbitrary linear combination of $C$ and $\sigma$, with coefficients depending on the spectral parameters, and give a classification of all solutions to the classical Yang-Baxter equation which are of this type (Theorem 5.1). The result has already been obtained in [1]: essentially, we slightly extend the proof given there in order to allow for solutions with singularities.

Turning to the verification of the Jacobi identity, we first of all use the derivation rule for the Poisson bracket $\{\cdot, \cdot\}$ and the Jacobi identity for the commutator $[\cdot, \cdot]$ to derive, from Eq. (79), the following relation $[2,14]$ :

$$
\begin{aligned}
& \left\{D_{k},\left\{D_{l}, D_{m}\right\}\right\}+\text { cyclic } \\
& \quad=\left[D_{k}, \mathrm{YB}(d)_{k l m}+\left\{D_{l}, d_{k m}\right\}-\left\{D_{m}, d_{k l}\right\}\right]+\text { cyclic } .
\end{aligned}
$$

Inserting Eq. (80), this becomes

$$
\begin{aligned}
& \left\{D_{k},\left\{D_{l}, D_{m}\right\}\right\}+\text { cyclic } \\
& \quad=\left[D_{k}, \mathrm{YB}(d)_{k l m}+\left[c_{l k}+c_{l m}, d_{k m}\right]-\left[c_{m k}+c_{m l}, d_{k l}\right]\right]+\text { cyclic } .
\end{aligned}
$$

Thus the rhs of this expression must vanish in order for the Jacobi identity to be satisfied. But actually, more than this is true: namely, we have

$$
\mathrm{YB}(d)_{k l m}+\left\{D_{l}, d_{k m}\right\}-\left\{D_{m}, d_{k l}\right\}=0,
$$


or equivalently,

$$
\mathrm{YB}(d)_{k l m}+\left[c_{l k}+c_{l m}, d_{k m}\right]-\left[c_{m k}+c_{m l}, d_{k l}\right]=0,
$$

as can be checked by an explicit calculation using the definitions (54) and (82) of $c$ and $d$, together with the identities (69)-(71) [cf. Eq. (106) in the appendix]: this confirms, for the class of models under consideration here, the validity of the "extended dynamical Yang-Baxter relation" postulated by Maillet [14]. [Indeed, if we write, as in Eq. (2.19) there,

$$
\begin{aligned}
& \left\{D_{k}\left(x_{k}, \lambda_{k}\right), d_{l m}\left(x_{l}, \lambda_{l} ; x_{m}, \lambda_{m}\right)\right\} \\
& \quad=\left\{L_{k}\left(x_{k}, \lambda_{k}\right),(s+r)_{m l}\left(x_{m} ; \lambda_{m}, \lambda_{l}\right)\right\} \delta\left(x_{l}-x_{m}\right) \\
& \quad=-H_{k, m l}\left(x_{k} ; \lambda_{k}, \lambda_{m}, \lambda_{l}\right) \delta\left(x_{k}-x_{m}\right) \delta\left(x_{l}-x_{m}\right),
\end{aligned}
$$

and recall that our $L$ corresponds to $-L$ there, we see that our Eq. (87), after splitting off a factor $\delta\left(x_{k}-x_{l}\right) \delta\left(x_{k}-x_{m}\right)$, becomes identical with Eq. (2.18) there.] Similarly, using once again the derivation rule for the Poisson bracket $\{\cdot, \cdot\}$ and the Jacobi identity for the commutator $[\cdot, \cdot]$, we obtain from Eqs. (80)-(81)

$$
\begin{aligned}
& \left\{D_{k},\left\{D_{l}, d_{m n}\right\}\right\}+\left\{D_{l},\left\{d_{m n}, D_{k}\right\}\right\}+\left\{d_{m n},\left\{D_{k}, D_{l}\right\}\right\} \\
& \quad=\left[d_{m n},\left[c_{k m}+c_{k n}, c_{l m}+c_{l n}\right]-\left[c_{k m}+c_{k n}, d_{k l}\right]+\left[c_{l m}+c_{l n}, d_{l k}\right]\right] .
\end{aligned}
$$

Again the rhs of this expression must vanish in order for the Jacobi identity to be satisfied, and again this can be checked by an explicit calculation using the definitions (54) and (82) of $c$ and $d$, together with the identities (69)-(72) and

$$
\begin{aligned}
{\left[\left[C_{k m}+C_{k n}, \sigma_{k l}\right], C_{m n}\right] } & =\left[\left[C_{l m}+C_{l n}, \sigma_{k l}\right], C_{m n}\right]=0, \\
{\left[\left[C_{k m}-C_{l n}, \sigma_{k l}\right], \sigma_{m n}\right] } & =\left[\left[C_{k n}-C_{l m}, \sigma_{k l}\right], \sigma_{m n}\right]=0,
\end{aligned}
$$

the second of which holds because

$$
\left[\left[C_{k m}, \sigma_{k l}\right], \sigma_{m n}\right]=-\left[\left[\sigma_{k n}, \sigma_{m n}\right], \sigma_{k l}\right]=\left[\left[C_{l n}, \sigma_{k l}\right], \sigma_{m n}\right] .
$$

Finally, in the remaining combinations

$$
\left\{D_{i},\left\{d_{k l}, d_{m n}\right\}\right\}+\left\{d_{k l},\left\{d_{m n}, D_{i}\right\}\right\}+\left\{d_{m n},\left\{D_{i}, d_{k l}\right\}\right\}
$$

and

$$
\left\{d_{i \jmath},\left\{d_{k l}, d_{m n}\right\}\right\}+\left\{d_{k l},\left\{d_{m n}, d_{i j}\right\}\right\}+\left\{d_{m n},\left\{d_{i \jmath}, d_{k l}\right\}\right\}
$$

each term vanishes separately.

Using the c's and d's, we can write down a closed algebra not only for the Lax operators $D(x, \lambda)$ but also for the transition matrices $T(x, y, \lambda)$ - at least when the relations involved are viewed as equations between distributions in all spatial variables that appear, so that no regularization is required. To this end, we continue to use the tensor notation introduced above, which - in view of the fact that the transition matrices take values in the Lie group $G$ - we can do provided we replace the universal enveloping algebra $U(\mathbf{g})$ by a "complete" algebra $\hat{U}(\mathbf{g})$ which incorporates both $U(\mathbf{g})$ and $G$ : this can be achieved by representing $U(\mathbf{g})$ as the algebra of left invariant differential operators $[10$, p. 108] and $G$ as the group of left translation operators on the space $C^{\infty}(G)$ of real-valued smooth functions on $G: \hat{U}(\mathbf{g})$ can then be defined as the subalgebra of linear operators on $C^{\infty}(G)$ generated by $U(\mathbf{g})$ and $G$. With this 
notation, the Poisson bracket relation (74) between the $L$ 's and the $j$ 's gives rise to a Poisson bracket relation between the T's and the $j$ 's, according to

$$
\begin{aligned}
& \left\{T_{k}(x, y, \lambda), j_{l m}(z)\right\} \\
& \quad=\int d z^{\prime} \varepsilon(x-y) \chi\left(z^{\prime} ; x, y\right) T_{k}\left(x, z^{\prime}, \lambda\right)\left\{L_{k}\left(z^{\prime}, \lambda\right), j_{l m}(z)\right\} T_{k}\left(z^{\prime}, y, \lambda\right),
\end{aligned}
$$

[cf. Eqs. (59), (60), (62)], namely

$$
\begin{aligned}
& \left\{T_{k}(x, y, \lambda), j_{l m}(z)\right\} \\
& \quad=\frac{2 \lambda}{1-\lambda^{2}} \varepsilon(x-y) \chi(z ; x, y) T_{k}(x, z, \lambda)\left[C_{k l}+C_{k m}, j_{l m}(z)\right] T_{k}(z, y, \lambda) .
\end{aligned}
$$

Observe again that due to the identity (69), this equation continues to hold when $j$ is replaced by $\sigma$ or by any other linear combination of $C$ and $j$, such as $r, s$, or $d$. Therefore, the basic Poisson brackets for the T's and d's [cf. (9), (53), (54)] take the following form:

$$
\begin{aligned}
\left\{T_{k}\left(x_{k}, y_{k}, \lambda_{k}\right), T_{l}\left(x_{l}, y_{l}, \lambda_{l}\right)\right\} & \\
= & +\varepsilon\left(x_{k}-y_{k}\right) \chi_{k l}\left(z ; x_{k}, y_{k}\right) \\
& \times\left. T_{k}\left(x_{k}, z, \lambda_{k}\right) T_{l}\left(x_{l}, z, \lambda_{l}\right) d_{l k}\left(z, \lambda_{l}, \lambda_{k}\right) T_{k}\left(z, y_{k}, \lambda_{k}\right) T_{l}\left(z, y_{l}, \lambda_{l}\right)\right|_{z=y_{l}} ^{z=x_{l}} \\
- & \varepsilon\left(x_{l}-y_{l}\right) \chi_{l k}\left(z ; x_{l}, y_{l}\right) \\
& \left.\times T_{k}\left(x_{k}, z, \lambda_{k}\right) T_{l}\left(x_{l}, z, \lambda_{l}\right)\right)\left.d_{k l}\left(z, \lambda_{k}, \lambda_{l}\right) T_{k}\left(z, y_{k}, \lambda_{k}\right) T_{l}\left(z, y_{l}, \lambda_{l}\right)\right|_{z=y_{k}} ^{z=x_{k}}, \\
\left\{T_{k}\left(x, y, \lambda_{k}\right), d_{l m}\left(z, \lambda_{l}, \lambda_{m}\right)\right\}= & \varepsilon(x-y) \chi_{k l m}(z ; x, y) T_{k}\left(x, z, \lambda_{k}\right)\left[c_{k l}\left(z, \lambda_{k}, \lambda_{l}\right)\right. \\
& \left.+c_{k m}\left(z, \lambda_{k}, \lambda_{m}\right), d_{l m}\left(z, \lambda_{l}, \lambda_{m}\right)\right] T_{k}\left(z, y, \lambda_{k}\right) .
\end{aligned}
$$

As before, the $\chi$ 's are characteristic functions of the respective intervals, their values at the points of discontinuity being allowed to depend on the spectral parameters involved (but not on the field $\varphi$ ):

$$
\begin{gathered}
\chi_{k l}(z ; x, y)= \begin{cases}\alpha_{k l} \equiv \alpha\left(\lambda_{k}, \lambda_{l}\right) & \text { for } z=\min \{x, y\} \\
1 & \text { for } \min \{x, y\}<z<\max \{x, y\} \\
\beta_{k l} \equiv \beta\left(\lambda_{k}, \lambda_{l}\right) & \text { for } z=\max \{x, y\} \\
0 & \text { otherwise }\end{cases} \\
\chi_{k l m}(z ; x, y)=\left\{\begin{array}{ll}
\alpha_{k l m} \equiv \alpha\left(\lambda_{k}, \lambda_{l}, \lambda_{m}\right) & \text { for } z=\min \{x, y\} \\
1 & \text { for } \min \{x, y\}<z<\max \{x, y\} \\
\beta_{k l m} \equiv \beta\left(\lambda_{k}, \lambda_{l}, \lambda_{m}\right) & \text { for } z=\max \{x, y\} \\
0 & \text { otherwise }
\end{array} .\right.
\end{gathered}
$$

As mentioned in the previous section, the values of the $\alpha$ 's and $\beta$ 's are irrelevant as long as (95) and (96) are interpreted as equations between distributions. Moreover, we can use the same reasoning as in [14] to conclude from Eqs. (95) and (96) that the Jacobi identity holds, in the sense of distributions, for the algebra generated by the T's and d's: in particular, it is valid for multiple Poisson brackets $\left\{T_{k}\left(x_{k}, y_{k}, \lambda_{k}\right),\left\{T_{l}\left(x_{l}, y_{l}, \lambda_{l}\right), \ldots\right\}\right\}$.

On the other hand, we recall from the previous section that fixing the values of the $\alpha$ 's and $\beta$ 's in Eqs. (97), (98) amounts to a specific choice of regularization for the Poisson brackets in the equal points limit $\left[x_{l}=x_{k}\right.$ or $x_{l}=y_{k}$ or $y_{l}=x_{k}$ or $y_{l}=y_{k}$ in (95), $z=x$ or $z=y$ in (96)], which is required to define Poisson brackets between 
transition matrices for coinciding or adjacent intervals, and in particular between monodromy matrices. Their choice is constrained by the derivation rule (68), which leads to

$$
\alpha_{k l}+\beta_{k l}=1, \quad \alpha_{k l m}+\beta_{k l m}=1
$$

Now since the regularized basic Poisson brackets between $T$ 's and $d$ 's are given by products of $T$ 's and $d$ 's, we are able to compute their multiple Poisson brackets from repeated application of the derivation rule and the regularized basic Poisson brackets. We shall refer to such a procedure as a one-step regularization - in contrast, e.g., to Maillet's "total symmetrization rule" [14] which is a multi-step regularization insofar as each multiple Poisson bracket requires a separate regularization that cannot be reduced to the regularization of multiple Poisson brackets with a smaller number of factors.

In order to see whether the above one-step regularization works, we compute the lhs of the Jacobi identity for three transition matrices on coinciding intervals. To begin with, we write down the regularized Poisson bracket of two transition matrices on coinciding intervals, assuming for simplicity that $x>y$ :

$$
\begin{aligned}
\left\{T_{k}\left(x, y, \lambda_{k}\right), T_{l}\left(x, y, \lambda_{l}\right)\right\}= & b_{k l}\left(x, \lambda_{k}, \lambda_{l}\right) T_{k}\left(x, y, \lambda_{k}\right) T_{l}\left(x, y, \lambda_{l}\right) \\
& -T_{k}\left(x, y, \lambda_{k}\right) T_{l}\left(x, y, \lambda_{l}\right) a_{k l}\left(y, \lambda_{k}, \lambda_{l}\right)
\end{aligned}
$$

with

$$
\begin{aligned}
& a_{k l}\left(y, \lambda_{k}, \lambda_{l}\right)=\left(\alpha_{k l}+\alpha_{l k}\right) r_{k l}\left(y, \lambda_{k}, \lambda_{l}\right)+\left(\alpha_{k l}-\alpha_{l k}\right) s_{k l}\left(y, \lambda_{k}, \lambda_{l}\right), \\
& b_{k l}\left(x, \lambda_{k}, \lambda_{l}\right)=\left(\beta_{k l}+\beta_{l k}\right) r_{k l}\left(x, \lambda_{k}, \lambda_{l}\right)+\left(\beta_{k l}-\beta_{l k}\right) s_{k l}\left(x, \lambda_{k}, \lambda_{l}\right) .
\end{aligned}
$$

[Note that in the special case $\alpha_{k l}=\beta_{k l}=\frac{1}{2}$, which corresponds to Mailet's "total symmetrization rule", Eq. (100) with Eq. (101) reduces to Eq. (64).] Now straightforward calculation gives

$$
\begin{aligned}
&\left\{T_{k}\left(x, y, \lambda_{k}\right),\left\{T_{l}\left(x, y, \lambda_{l}\right), T_{m}\left(x, y, \lambda_{m}\right)\right\}\right\}+\text { cyclic } \\
&=+T_{k}\left(x, y, \lambda_{k}\right) T_{l}\left(x, y, \lambda_{l}\right) T_{m}\left(x, y, \lambda_{m}\right) \\
& \times\left\{\operatorname{YB}(a(y))_{k l m}+\left(\frac{2 \lambda_{k}}{1-\lambda_{k}^{2}}\left[C_{k l}+C_{k m}, \alpha_{k l m} a_{l m}\left(y, \lambda_{l}, \lambda_{m}\right)\right]+\text { cyclic }\right)\right\} \\
&-\left\{\operatorname{YB}(b(x))_{k l m}-\left(\frac{2 \lambda_{k}}{1-\lambda_{k}^{2}}\left[C_{k l}+C_{k m}, \beta_{k l m} b_{l m}\left(x, \lambda_{l}, \lambda_{m}\right)\right]+\text { cyclic }\right)\right\} \\
& \times T_{k}\left(x, y, \lambda_{k}\right) T_{l}\left(x, y, \lambda_{l}\right) T_{m}\left(x, y, \lambda_{m}\right) .
\end{aligned}
$$

It is hard to see how the rhs of this equation can be zero, identically in $x, y, \varphi$, and $\dot{\varphi}$, unless the terms in curly brackets vanish separately. This, however, is excluded by the following no-go theorem:

Theorem 4.1. With the above notation, there is no choice for the functions $\alpha_{k l}, \alpha_{k l m}$ and $\beta_{k l}, \beta_{k l m}$, subject to the condition (99), such that the two terms in curly brackets of Eq. (102) vanish separately.

A proof of this theorem will be given in the appendix. 


\section{Appendix}

Our purpose in this appendix is to formulate and prove the classification theorem mentioned in Sect. 4 and to present a proof of the no-go-theorem given at the end of Sect. 4.

\subsection{Classification Theorem}

The classification theorem concerns solutions $t$ of the classical Yang-Baxter equation $^{8}$

$$
\mathrm{YB}(t)_{k l m}=0
$$

where, by definition,

$$
\mathrm{YB}(t)_{k l m}=\left[t_{k l}, t_{k m}\right]+\left[t_{k l}, t_{l m}\right]-\left[t_{k m}, t_{m l}\right]
$$

and where $t$ is supposed to be a linear combination of the Casimir tensor $C$ for $\mathbf{g}$ and some fixed involutive automorphism $\sigma$ of $\mathbf{g}$,

$$
t=a C+b \sigma .
$$

Note that, due to the identities (69)-(71), we have

$$
\begin{aligned}
\mathrm{YB}(t)_{k l m}= & \left(a_{k l} a_{k m}-a_{k l} a_{l m}-a_{k m} a_{m l}\right)\left[C_{k l}, C_{k m}\right] \\
& +\left(a_{k l} b_{k m}-a_{k l} b_{l m}-b_{k m} b_{m l}\right)\left[C_{k l}, \sigma_{k m}\right] \\
& -\left(a_{k m} b_{k l}-a_{k m} b_{m l}-b_{k l} b_{l m}\right)\left[C_{k m}, \sigma_{k l}\right] \\
& -\left(a_{l m} b_{k l}+a_{m l} b_{k m}-b_{k l} b_{k m}\right)\left[C_{l m}, \sigma_{k l}\right] .
\end{aligned}
$$

As a further input, we must specify what kind of functions of the spectral parameters we admit for the coefficients $a$ and $b$ in Eq. (105).

To this end, we first of all set $\mathscr{R}_{0}=\mathbb{K}$ (the ground field $\mathbb{K}$ will for simplicity be supposed to be either $\mathbb{R}$ or $\mathbb{C}$ ) and, for any integer $n \geq 1$, we let $\mathscr{R}_{n}$ denote the ring of $\mathbb{K}$-valued functions $f$ defined on open, dense domains $D_{f}$ in $\mathbb{K}^{n}$, i.e.,

$$
\mathscr{R}_{n}=\left\{\left(f, D_{f}\right) \mid D_{f} \text { is open and dense in } \mathbb{K}^{n}, f: D_{f} \rightarrow \mathbb{K}\right\} \text {. }
$$

Of course, addition and multiplication in $\mathscr{B}_{n}$ are defined as usual, i.e., pointwise,

$$
\begin{aligned}
\left(f, D_{f}\right)+\left(g, D_{g}\right) & =\left(\left.f\right|_{D_{f} \cap D_{g}}+\left.g\right|_{D_{f} \cap D_{g}}, D_{f} \cap D_{g}\right), \\
\left(f, D_{f}\right) \cdot\left(g, D_{g}\right) & =\left(\left.\left.f\right|_{D_{f} \cap D_{g}} \cdot g\right|_{D_{f} \cap D_{g}}, D_{f} \cap D_{g}\right),
\end{aligned}
$$

which makes sense because, in any topological space, the intersection of two open dense subsets is again open and dense. Next, let $\left(R_{n}\right)_{n \geq 0}$ be a family of subrings $R_{n}$ of $\mathscr{\ell}_{n}$ containing the constant functions, with $R_{0}=\mathbb{K}$, which is closed under division as well as under extension and restriction of variables, i.e., satisfies the following conditions:

(a) Each $R_{n}$ is a division ring, i.e., every non-zero element $\left(f, D_{f}\right)$ in $R_{n}$ has an inverse $\left(f^{-1}, D_{f^{-1}}\right)$ in $R_{n}$. Explicitly, this means that for every non-zero $\left(f, D_{f}\right) \in$ $R_{n}$,

$\left(\mathrm{a}_{1}\right) D_{f-1}=\left\{\lambda \in D_{f} \mid f(\lambda) \neq 0\right\}$ is open and dense in $\mathbb{K}^{n}$,

$\left(\mathrm{a}_{2}\right)\left(f^{-1}, D_{f-1}\right) \in R_{n}$.

\footnotetext{
8 We continue to use the tensor notation introduced in Sect. 4
} 
(b) For $\left(f, D_{f}\right)$ in $R_{n}$ and $1 \leq k \leq n$, the extension $\left(f_{(k)}, D_{f_{(k)}}\right)$, defined by

$$
\begin{aligned}
D_{f_{(k)}}=\{ & \left.\left(\lambda_{1}, \ldots, \lambda_{n+1}\right) \in \mathbb{K}^{n+1} \mid\left(\lambda_{1}, \ldots, \lambda_{k-1}, \lambda_{k+1}, \ldots, \lambda_{n+1}\right) \in D_{f}\right\} \\
& f_{(k)}\left(\lambda_{1}, \ldots, \lambda_{n+1}\right)=f\left(\lambda_{1}, \ldots, \lambda_{k-1}, \lambda_{k+1}, \ldots, \lambda_{n+1}\right)
\end{aligned}
$$

is in $R_{n+1}$; more precisely, this is the extension which is constant in the $k^{\text {th }}$ variable (c) For $\left(f, D_{f}\right)$ in $R_{n}$ and $1 \leq k \leq n-1$ and for any number $\zeta$ in $\operatorname{pr}_{k}\left(D_{f}\right) \subset \mathbb{k}$ $\left(\mathrm{pr}_{k}\right.$ denotes the $k^{\text {th }}$ projection from $\mathbb{K}^{n}$ to $\left.\mathbb{K}: \operatorname{pr}_{k}\left(\lambda_{1}, \ldots, \lambda_{n}\right)=\lambda_{k}\right)$, the restriction $\left(f_{\zeta}^{(k)}, D_{f_{\zeta}^{(k)}}\right)$, defined by

$$
\begin{gathered}
D_{f_{\zeta}^{(k)}}=\left\{\left(\lambda_{1}, \ldots, \lambda_{n-1}\right) \in \mathbb{K}^{n-1} \mid\left(\lambda_{1}, \ldots, \lambda_{k-1}, \zeta, \lambda_{k+1}, \ldots, \lambda_{n-1}\right) \in D_{f}\right\}, \\
f_{\zeta}^{(k)}\left(\lambda_{1}, \ldots, \lambda_{n-1}\right)=f\left(\lambda_{1}, \ldots, \lambda_{k-1}, \zeta, \lambda_{k+1}, \ldots, \lambda_{n-1}\right),
\end{gathered}
$$

is in $R_{n-1}$; in particular, this requires $D_{f_{\zeta}^{(k)}}$ to be open and dense in $\mathbb{K}^{n-1}$. It shoul also be noted that the set $\operatorname{pr}_{k}\left(D_{f}\right)$ of admissible $\zeta$ 's is itself open and dense in $\mathbb{K}$ because $\mathrm{pr}_{k}$ is continuous, open, and onto.

Important examples for such families are rings of rational functions and rings o meromorphic functions in several complex variables.

Note that repeated application of the extension property (b) above leads to variou embeddings of $R_{m}$ into $R_{n}$, as soon as $m \leq n$. In particular, we have the embedding

$$
R_{1} \rightarrow R_{n}, \quad f \mapsto f_{k} \quad\left(f_{k}\left(\lambda_{1}, \ldots, \lambda_{n}\right)=f\left(\lambda_{k}\right)\right)
$$

for $1 \leq k \leq n$ and

$$
R_{2} \rightarrow R_{n}, \quad f \mapsto f_{k l} \quad\left(f_{k l}\left(\lambda_{1}, \ldots, \lambda_{n}\right)=f\left(\lambda_{k}, \lambda_{l}\right)\right)
$$

for $1 \leq k, l \leq n$. With this notation (which is analogous to that introduced at th beginning of Sect.4), we are ready to formulate the classifcation theorem:

Theorem 5.1. Let $\left(R_{n}\right)_{n \geq 0}$ be a family of function rings satisfying the conditions give. above. Let $a$ and $b$ be in $R_{2}$. Then $t=a C+b \sigma$ satisfies the classical Yang-Baxte equation (103) if and only if there exist a function $g \in R_{1}$ and a non-constant functio. $f \in R_{1}$ such that $t$ takes one of the following forms:

$$
\begin{gathered}
t_{k l}=g_{l} \frac{C_{k l}}{f_{k}-f_{l}}, \\
t_{k l}=g_{l} \frac{C_{k l}+\sigma_{k l}}{f_{k}-f_{l}} \\
t_{k l}=g_{l}\left(\frac{C_{k l}}{f_{k}-f_{l}}-\frac{\sigma_{k l}}{f_{k}+f_{l}}\right) .
\end{gathered}
$$

If in addition $t$ is antisymmetric, it is of the form i) or ii), with $g$ constant.

Proof. Looking at Eq. (104) we see that if $t_{k l}$ is a solution of the classical Yang-Baxte equation (103), then so is any multiple $g_{l} t_{k l}$. Hence using the identities (69)-(71), i is easily verified that the formulas given above do provide solutions of Eq. (103). 
Conversely, suppose now that $t$ satisfies the classical Yang-Baxter equation (103). As the four commutators appearing in Eq. (106) are linearly independent, we obtain four equations on the coefficients $a$ and $b$ :

$$
\begin{aligned}
a_{k l} a_{k m}-a_{k l} a_{l m}-a_{k m} a_{m l} & =0, \\
a_{k l} b_{k m}-a_{k l} b_{l m}-b_{k m} b_{m l} & =0, \\
a_{k m} b_{k l}-a_{k m} b_{m l}-b_{k l} b_{l m} & =0, \\
a_{l m} b_{k l}+a_{m l} b_{k m}-b_{k l} b_{k m} & =0 .
\end{aligned}
$$

Now if $a=0$, Eqs. (108)-(110) imply $b_{k l} b_{k m}=b_{k l} b_{l m}=b_{k m} b_{m l}=0$ and hence $b=0$, because $R_{n}$, being a division ring, has no zero divisors. Thus we get only the trivial solution $t=0$, contained in any of the above cases $(g=0)$. We may therefore assume without loss of generality that $a \neq 0$ and divide Eq. (107) by $a_{k l} a_{k m} a_{l m}$ to obtain

$$
\frac{1}{a_{k m}}=\frac{1}{a_{l m}}-\frac{a_{m l}}{a_{l m}} \frac{1}{a_{k l}} .
$$

The lhs of this equation does not depend on $\lambda_{l}$. Hence choosing a fixed number $\zeta$ in $\operatorname{pr}_{1}\left(D_{a}\right) \cap \operatorname{pr}_{2}\left(D_{a}\right)$ (a set which, as noted in condition (c) above, is the intersection of two open dense subsets of $\mathbb{K}$ and hence is again open and dense in $\mathbb{K}$ ) and setting

$$
r(\lambda)=a(\zeta, \lambda), \quad s(\lambda)=-\frac{a(\lambda, \zeta)}{a(\zeta, \lambda)}, \quad f(\lambda)=\frac{1}{a(\lambda, \zeta)},
$$

we obtain

$$
\frac{1}{a_{k m}}=r_{m}^{-1}+s_{m} f_{k} \quad \text { and } \quad \frac{1}{a_{k l}}=r_{l}^{-1}+s_{l} f_{k} .
$$

Inserting this into Eq. (111) we infer that

$$
a_{l m} r_{m}^{-1}+a_{m l} r_{l}^{-1}-1=-\left(a_{l m} s_{m}+a_{m l} s_{l}\right) f_{k} .
$$

Suppose now that $a_{l m} s_{m}+a_{m l} s_{l} \neq 0$. Then dividing Eq. (113) by $a_{l m} s_{m}+a_{m l} s_{l}$, we would conclude that $f$ must be constant. But $f$ cannot be constant, because otherwise $a_{k l}$ would depend on its second argument $\lambda_{l}$ only, i.e., $a_{k l}=r_{l}$ with $r \in R_{1}$, and as a result, Eq. (107) would read

$$
0=r_{l} r_{m}-r_{l} r_{m}-r_{m} r_{l}=-r_{m} r_{l}
$$

implying, be the same argument as before (absence of zero divisors), that $a$ would have to vanish, contrary to the assumption. Hence $a_{l m} s_{m}+a_{m l} s_{l}=0$, which means that the rescaled coefficients $\tilde{a}_{l m}=a_{l m} s_{m}$ are antisymmetric: $\tilde{a}_{m l}=-\tilde{a}_{l m}$.

To summarize, we have shown that from the original solution $t$ of the classical Yang-Baxter equation (103) of the form (105), we obtian a new solution of that form, namely

$$
\tilde{t}_{k l}=t_{k l} s_{l}, \quad \tilde{a}_{k l}=a_{k l} s_{l}, \quad \tilde{b}_{k l}=b_{k l} s_{l},
$$

with the additional bonus that this new solution has antisymmetric $a$-coefficients:

$$
\tilde{a}_{l k}=-\tilde{a}_{k l} .
$$

In particular, this means that Eqs. (107)-(110) are also satisfied when the $a$ 's and $b$ 's are replaced by the $\tilde{a}$ 's and $\tilde{b}$ 's.

Now replacing the $a$ 's in Eq. (111) by $\tilde{a}$ 's we see that $\tilde{a}_{k m}^{-1}=\tilde{a}_{k l}^{-1}-\tilde{a}_{m l}^{-1}$, while inserting Eq. (114) in Eq. (112) then gives

$$
\tilde{a}_{k m}^{-1}=\tilde{a}_{k l}^{-1}-\tilde{a}_{m l}^{-1}=a_{k l}^{-1} s_{l}^{-1}-a_{m l}^{-1} s_{l}^{-1}=\left(r_{l}^{-1} s_{l}^{-1}+f_{k}\right)-\left(r_{l}^{-1} s_{l}^{-1}+f_{m}\right),
$$


i.e.,

$$
\tilde{a}_{k l}^{-1}=f_{k}-f_{l}
$$

Defining $g=s^{-1}$ we get

$$
a_{k l}=\frac{g_{l}}{f_{k}-f_{l}} .
$$

Thus if $b=0$, we arrive at the solution (i). Suppose therefore that $b \neq 0$. Dividing Eq. (110), with $a$ 's and $b$ 's replaced by $\tilde{a}$ 's and $\tilde{b}$ 's, by $\tilde{b}_{k l} \tilde{b}_{k m} \tilde{a}_{l m}$ gives

$$
\tilde{b}_{k l}^{-1}=\tilde{b}_{k m}^{-1}-\tilde{a}_{l m}^{-1}=\tilde{b}_{k m}^{-1}-f_{l}+f_{m},
$$

i.e.,

$$
\tilde{b}_{k l}^{-1}+f_{l}=\tilde{b}_{k m}^{-1}+f_{m} .
$$

Both sides of this equation can depend on $\lambda_{k}$ only (the lhs does not depend on $\lambda_{m}$ and the rhs does not depend on $\lambda_{l}$ ), so there must exist a function $h \in R_{1}$ such that

$$
\tilde{b}_{k l}^{-1}=h_{k}-f_{l} \text {. }
$$

Now dividing Eq. (109), with a's and $b$ 's replaced by $\tilde{a}$ 's and $\tilde{b}$ 's, by $\tilde{a}_{k m} \tilde{b}_{k l}$ and inserting Eqs. (115) and (117), we get

$$
\frac{h_{k}-f_{l}}{h_{m}-f_{l}}+\frac{f_{k}-f_{m}}{h_{l}-f_{m}}=1
$$

or

$$
\frac{h_{l}-f_{k}}{f_{l}-h_{k}}=\frac{h_{l}-f_{m}}{f_{l}-h_{m}} .
$$

Again, both sides of this last equation can depend on $\lambda_{l}$ only, so choosing two fixed numbers $\zeta$ and $\zeta^{\prime}$ in $D_{f} \cap D_{h}$ and setting $\alpha=f(\zeta), \beta=h(\zeta), \alpha^{\prime}=f\left(\zeta^{\prime}\right), \beta^{\prime}=h\left(\zeta^{\prime}\right)$, we see that Eq. (118), with $\lambda_{l}=\lambda, \lambda_{k}=\zeta, \lambda_{m}=\zeta^{\prime}$, becomes

$$
\left(\beta-\beta^{\prime}\right) h_{l}=\left(\alpha-\alpha^{\prime}\right) f_{l}+\alpha^{\prime} \beta-\alpha \beta^{\prime} .
$$

As $f$ is a non-constant function, we can choose $\zeta$ and $\zeta^{\prime}$ in such a way that $\alpha \neq \alpha^{\prime}$. But then, for the same reason, $\beta \neq \beta^{\prime}$, and hence $h$ is a non-constant function given by

$$
h=\gamma f+\delta,
$$

with

$$
\gamma=\frac{\alpha-\alpha^{\prime}}{\beta-\beta^{\prime}} \neq 0 \quad \text { and } \quad \delta=\frac{\alpha^{\prime} \beta-\alpha \beta^{\prime}}{\beta-\beta^{\prime}} .
$$

On the other hand, Eq. (119) taken at $\lambda=\zeta$ and at $\lambda=\zeta^{\prime}$ gives

$$
\beta=\gamma \alpha+\delta \text { and } \beta^{\prime}=\gamma \alpha^{\prime}+\delta .
$$

Eqs. (120) and (121) imply

$$
\gamma= \pm 1 \quad \text { and } \quad \delta(\gamma+1)=0,
$$

and hence either

$$
h= \pm f,
$$

or

$$
h=-f+\delta .
$$


The first case yields

$$
b_{k l}= \pm \frac{g_{l}}{f_{k} \mp f_{l}}
$$

i.e., solutions (ii) and (iii), whereas the second case results in

$$
b_{k l}=\frac{g_{l}}{\delta-f_{k}-f_{l}} .
$$

So shifting $f$ by $\delta / 2$ gives again (iii), q.e.d.

\subsection{Proof of the No-Go Theorem}

Here we shall prove the no-go theorem given at the end of Sect. 4.

Proof. Write the quantities $a_{k l}$ and $b_{k l}$ in Eq. (101) as linear combinations of $C_{k l}$ and $\sigma_{k l}$, i.e.,

$$
\begin{aligned}
& a_{k l}=f_{k l} C_{k l}+g_{k l} \sigma_{k l}, \\
& b_{k l}=\phi_{k l} C_{k l}+\gamma_{k l} \sigma_{k l},
\end{aligned}
$$

where the functions $f_{k l}, g_{k l}, \phi_{k l}$, and $\gamma_{k l}$ depend on $\lambda_{k}$ and $\lambda_{l}$ and are antisymmetric. Now looking at the expressions in curly brackets in Eq. (101), we see that the terms containing a commutator with $C_{k l}+C_{k m}$ (plus cyclic permutations) give rise to $C-\sigma$ type commutators but not to $C-C$ type commutators: the only contribution to the latter comes from the Yang-Baxter term. Thus according to Eq. (107), we get

$$
\begin{gathered}
f_{k l} f_{k m}-f_{k l} f_{l m}-f_{k m} f_{m l}=0, \\
\phi_{k l} \phi_{k m}-\phi_{k l} \phi_{l m}-\phi_{k m} \phi_{m l}=0 .
\end{gathered}
$$

Arguing as in the proof of Theorem 5.1 to derive Eq. (115) from Eq. (107), we conclude that there exist non-constant functions $f$ and $\phi$ of one real variable $\lambda$ and real numbers $\varepsilon_{1}$ and $\varepsilon_{2}$ which are either 0 or 1 , such that

$$
\begin{aligned}
f_{k l} & =\frac{\varepsilon_{1}}{f_{k}-f_{l}}, \\
\phi_{k l} & =\frac{\varepsilon_{2}}{\phi_{k}-\phi_{l}} .
\end{aligned}
$$

On the other hand, using (82), the $C_{k l}$-coefficients of $a_{k l}$ and $b_{k l}$ can be computed in terms of $\alpha_{k l}$, whence we have the following two equations:

$$
\begin{gathered}
\frac{\varepsilon_{1}}{f_{k}-f_{l}}=-\frac{1}{\lambda_{k}-\lambda_{l}}\left(\frac{2 \lambda_{k}^{2}}{1-\lambda_{k}^{2}} \alpha_{k l}+\frac{2 \lambda_{l}^{2}}{1-\lambda_{l}^{2}} \alpha_{l k}\right), \\
\frac{\varepsilon_{2}}{\phi_{k}-\phi_{l}}=\frac{1}{\lambda_{k}-\lambda_{l}}\left(\frac{2 \lambda_{k}^{2}}{1-\lambda_{k}^{2}}\left(\alpha_{k l}-1\right)+\frac{2 \lambda_{l}^{2}}{1-\lambda_{l}^{2}}\left(\alpha_{l k}-1\right)\right) .
\end{gathered}
$$

Adding the two equations gives the following $\alpha$-independent consistency condition:

$$
\frac{\varepsilon_{1}}{f_{k}-f_{l}}+\frac{\varepsilon_{2}}{\phi_{k}-\phi_{l}}=-\frac{1}{\lambda_{k}-\lambda_{l}}\left(\frac{2 \lambda_{k}^{2}}{1-\lambda_{k}^{2}}+\frac{2 \lambda_{l}^{2}}{1-\lambda_{l}^{2}}\right) \text {. }
$$

Now we use the following singularity argument. ${ }^{9}$ For $\lambda_{k}= \pm 1$, the rhs of this equation is singular for all values of $\lambda_{l}$, while the denominators on the lhs can vanish

9 We are indebted to C. Nowak for suggesting this simple reasoning 
only for certain values of $\lambda_{l}$ because the functions $f$ and $\phi$ are not constant. Therefore this last equation cannot be satisfied, and a contradiction is established, q.e.d.

Acknowledgements. We would like to thank C. Nowak and U. Pinkall for helpful discussions and pointing out ref. [3].

\section{References}

1. Avan, J., Talon, M.: Rational and trigonometric constant non-antisymmetric $R$-matrices. Phys. Lett. B 241, 77-82 (1990)

2. Babelon, O., Viallet, C.-M.: Hamiltonian structures and Lax equations. Phys. Lett. B 237, 411 $416(1990)$

3. Burstall, F.E., Ferus, D., Pedit, F., Pinkall, U.: Harmonic Tori in symmetric spaces and commuting Hamiltonian systems on loop algebras. University of Bath/Technische Universität Berlin/Emory University Atlanta, Preprint 1991

4. Vega, H.J. de, Eichenherr, H., Maillet, J.-M.: Classical and quantum algebras of non-local charges in sigma models. Commun. Math. Phys. 92, 507--524 (1984)

5. Eichenherr, H., Forger, M.: On the dual symmetry of the nonlinear sigma models. Nucl. Phys. B 155, 381-393 (1979)

More about nonlinear sigma models on symmetric spaces. Nucl. Phys. B 164, 528-535 (1980); B 282, 745-746 (1987) (Erratum)

Higher local conservation laws for nonlinear sigma models on symmetric spaces. Commun. Math. Phys. 82, 227-255 (1981)

6. Faddeev, L.D., Takhtajan, L.A.: Hamiltonian methods in the theory of solitons. Berlin Heidelberg New York: Springer 1987

7. Forger, M.: Nonlinear sigma models on symmettic spaces. In: Nonlinear partial differential operators and quantization procedures. Proceedings, Clausthal, Germany 1981, Andersson, S.I., Doebner, H.D. (eds.); Lect. Notes in Math. 1037. Berlin: Springer 1983

8. Forger, M., Laartz, J., Schäper, U.: Current algebra of classical non-linear sigma models. Commun. Math. Phys. 146, 397-402 (1992)

9. Freidel, L., Maillet, J.M.: Quadratic algebras and integrable systems. Preprint LPTHE-24/91; On classical and quantum integrable field theories associated to Kac-Moody current algebras. Preprint LPTHE-25/91

10. Helgason, S.: Differential geometry, Lie groups, and symmetric spaces. New York: Academic Press 1978

11. Laartz, J.: The extension structure of $2 D$ massive current algebras. Freiburg University preprint THEP 91/21, to be published in Mod. Phys. Lett. A

12. Maillet, J.-M.: Kac-Moody algebra and extended Yang-Baxter relations in the $O(N)$ non-linear sigma model. Phys. Lett. B 162, 137-142 (1985)

13. Maillet, J.-M.: Hamiltonian structures for integrable classical theories from graded Kac-Moody algebras. Phys. Lett. B 167, 401-405 (1986)

14. Maillet, J.-M.: New integrable canonical structures in two-dimensional models. Nucl. Phys. B 269, 54-76 (1986)

Communicated by N. Yu. Reshetikhin

Note added in proof. After completing the paper we have learned that for field independent nonsymmetric solutions of the CYBE a classification theorem involving more general automorphisms of the loop algebra of the spectral parameter exist $[1,2]$. overlapping partly with the result obtained in the appendix.

1. Avan, J., Talon, M.: Graded $R$-matrices for integrable systems. Nucl. Phys. B 352, 215 (1991)

2. Avan, J.: Current algebra realization of $R$-matrices associated to $Z_{2}$-graded Lie algebras. Phys. Lett. B 252, 230 (1990) 